\title{
Hommage à Pierre Lequien
}

\section{Tribute to Pierre Lequien}

\author{
A. Evrard \\ (C) Springer-Verlag France 2013
}

Lorsque j'ai rencontré Pierre Lequien en 2005, le dialogue entre professionnels de la périnatalité et usagers était encore embryonnaire. Il ne s'était instauré que dans quelques lieux où des professionnels pionniers avaient accepté de nous ouvrir leurs portes. Invitée à intervenir lors d'une journée du réseau Ombrel en 2006, j'ai compris que le Professeur Lequien faisait partie de ces soignants éclairés avec qui nous pouvions envisager un vrai travail de partenariat. Mes échanges avec lui furent d'emblée fluides, constructifs et privilégiés.

Convaincu de l'intérêt d'offrir une tribune à la parole des usagers, il fit partie de ceux qui initièrent notre participation aux Journées de la SFMP, d'abord à Marseille en 2007 puis à Deauville. Il nous donna ainsi l'occasion de formuler nos positions pour un public de professionnels pas toujours acquis à notre cause et ce fut très structurant.

En nous sollicitant pour collaborer, dès sa création, à la Revue de médecine périnatale, il nous permis de franchir une nouvelle étape. J'ai été bien sûr tout de suite intéressée mais dans le même temps, quelque peu inquiète. Sa confiance, son accueil, son souci de nous intégrer réellement au comité de rédaction balayèrent mes doutes. Il sut donner à ce projet un élan dynamique, stimulant et exigeant.

Pour nous, représentants d'usagers, c'était un lieu inespéré pour publier nos analyses et il défendit nos articles chaque fois que ce fut nécessaire, en valorisant toujours notre apport. En nous associant à une telle aventure, il nous offrait aussi un formidable lieu de formation et d'apprentissage. Et j'ai énormément appris à son contact. La rigueur de ses positions, son regard sur l'évolution de la périnatalité, son ouverture d'esprit et sa curiosité toujours exigeante ont été pour moi autant d'aiguillons pour mieux appréhender ce monde professionnel auquel je n'appartenais pas.

On ne peut oublier sa présence chaleureuse, sa joie et son humour toujours présents, son plaisir manifeste à explorer de nouvelles pistes et à préparer l'avenir. Je savais qu'ils travaillaient avec quelques autres à ouvrir le Conseil d'Administration de la SFMP aux représentants d'usagers. C'est avec quelques évocations pleines de facétie qu'il nous fit comprendre que ce n'était pas toujours simple! Pourtant, en octobre dernier, à Montpellier, j'ai participé pour la première fois à cette instance, malheureusement en l'absence de Pierre Lequien. J'ai eu le tort de ne pas lui communiquer tout de suite mes premières impressions et je regrette de ne pas avoir pu les partager avec lui et bénéficier de son éclairage.

C'est avec humilité que j'écris aujourd'hui ces lignes car ceux qui ont travaillé quotidiennement avec lui peuvent l'évoquer plus et mieux que moi. Je voulais toutefois lui rendre hommage, personnellement d'abord, car je ne serais pas la militante que je suis aujourd'hui sans son apport. Et aussi parce que parmi ses nombreuses réalisations, il a cru et participé à une nouvelle approche des relations usagerssoignants, témoignant de sa grande pertinence à envisager l'avenir.

A. Evrard $(\bowtie)$

Association Bien Naître, Collectif interassociatif autour de la naissance, 101, rue Pierre-Corneille, F-69003 Lyon, France e-mail : aevrard.pbuchou@wanadoo.fr 